$63^{\text {ème }}$ Congrès de la SFCO, 02014 (2015)

DOI:10.1051/sfco/20156302014

(c) Owned by the authors, published by EDP Sciences, 2015

\title{
COMMUNICATION
}

\section{Fibrome ossifiant juvénile : discussion diagnostique et thérapeutique à propos d'un cas}

Fenelon M, Jalbert F, Lauwers F

Service de Chirurgie Maxillo-faciale, CHU Purpan, Toulouse

Les tumeurs mandibulaires pédiatriques sont rares et se manifestent le plus souvent par une tuméfaction jugale ou elles sont découvertes fortuitement suite à la réalisation d'une imagerie (Perry \& al. 2014). Le Fibrome Ossifiant Juvénile (FOJ) se distingue des autres lésions osseuses fibromateuses par l'âge au quel il apparaît ainsi que par son comportement clinique (Williams \& al. 2000). On différencie la forme psammomatoides de la forme trabéculaire, cette dernière affectant préférentiellementla mandibule (El Mofty 2002, Barnes \& al. 2005). Cliniquement et histologiquement le FOJ peut parfois être confondu avec une tumeur maligne en raison d'une croissance rapide caractérisée par la présence de mitoses à l'examen microscopique (Slootweg 2009, Odin \& al. 2012). Le FOJ est plus infiltrant que le fibrome ossifiant retrouvé chez l'adulte (Perry \& al. 2014). Ainsi, il s'agit d'une tumeur localement agressive dont les récidives sont fréquentes (Odin \& al. 2012), rendant sa prise en charge complexe.

Un enfant de 2 ans $1 / 2$ était vu en consultation au sein du service de Chirurgie Maxillo faciale de Toulouse pour une tuméfaction jugale droite apparue depuis un mois. Cet enfant ne présentait pas de problème de santé particulier. On ne notait pas d'antécédent de traumatisme ou d'infection. L'examen clinique révélait un œdème jugal droit et il n’y avait pas d'adénopathie à la palpation. L'examen endo buccale mettait en évidence une voussure osseuse mandibulaire droite avec une muqueuse de recouvrement saine. L'examen tomodensitométrique objectivait une lésion de $4 \mathrm{~cm}$ de grand axe au niveau de l'angle mandibulaire et du ramus droits. Il s'agissait d'une lésion d'allure mixte soufflant les corticales vestibulaire et linguale qui étaient interrompues par endroit. L'examen anatomo pathologique pratiqué suite à une biopsie de la lésion évoquait un FOJ. Il a été décidé de réaliser une exérèse complète de la lésion avec dissection du nerf alvéolaire inférieur, et un blocage inter maxillaire a été laissé en place deux semaines (afin de limiter le risque fracturaire). A 3 mois, le conebeam montrait une ré-ossification quasi complète et l’œdème jugal avait régressé.

Le FOJ est une tumeur bénigne localement agressive d'évolution rapide (Ordin \& al. 2012). Selon la taille de la lésion ou l'atteinte du bord basilaire, certains auteurs recommandent d'avoir recours d'emblée à une chirurgie interruptrice (Dominguete 2007, Hemavathy \& Roy 2011). Notre patient présentait une lésion volumineuse de $4 \mathrm{~cm}$ de grand axe avec effraction par endroits des corticales vestibulaire et linguale. Malgré le risque fracturaire, il a été décidé de réaliser un traitement conservateur par curetage de la lésion car le jeune âge du patient et la conservation de l'intégrité du périoste laissaient envisager un fort potentiel ostéogénique. En effet, un fort potentiel de réparation osseuse lié au périoste existe chez l'enfant (Malizos \& Papatheodorou 2005). Ainsi une régénération osseuse spontanée a été rapportée dans les limites d'une résection interruptrice réalisée pour un FOJ mandibulaire chez un enfant de 7 ans (Espinosa \& al. 2006). Néanmoins, les récurrences sont fréquentes justifiant parfois le recours à une chirurgie interruptrice en seconde intention (Abuzinada \& Alyamani 2009, Perry \& al. 2014).

This is an Open Access article distributed under the terms of the Creative Commons Attribution License 4.0, which permits unrestricted use, distribution, and reproduction in any medium, provided the original work is properly cited. 
$63^{\text {ème }}$ Congrès de la SFCO

Nom et adresse du conférencier

Mathilde FENELON

CHU Toulouse

Place du Docteur Joseph Baylac

31300 Toulouse (France)

mathildefenelon@live.fr 